\title{
Searches for highly ionizing particles in ATLAS and CMS
}

\author{
Antonio Policicchio*† \\ on behalf of ATLAS and CMS collaborations \\ E-mail: antonio.policicchiodcern.ch
}

Latest searches of the ATLAS and the CMS experiments at LHC are presented for signatures with anomalous energy loss by ionization and/or small time-of-flight. The results are interpreted in terms of the production of new particles with a fractional or multiple value of the charge of the electron, the production of high mass stable charged particles, the presence of magnetic monopoles that lead to unusual ionization interactions with matter or the delayed effect of particles stopping in the detector volume. The signatures are also reinterpreted in the context of supersymmetric scenarios that predict stable or pseudo-stable charged particles in the final state.

The European Physical Society Conference on High Energy Physics

22-29 July 2015

Vienna, Austria

* Speaker.

†INFN Cosenza and CERN 


\section{Introduction}

Many extensions of the standard model (SM) include heavy, long-lived, charged particles that have speed significantly smaller than the speed of light $[1,2,3]$ or charge $Q$ not equal to the elementary positive or negative charge $\pm 1 \mathrm{e}[4,5,6,7,8]$, or both. With lifetimes greater than a few nanoseconds, these particles can travel distances comparable to the size of the ATLAS [9] and CMS [10] detectors at the Large Hadron Collider (LHC) and thus appear to be stable. These particles, generically referred to as heavy stable charged particles (HSCP), can be singly charged $(|Q|=1 e$ ), fractionally charged $(|Q|<1 e)$, or multiply charged $(|Q|>1 e)$. Without dedicated searches, HSCPs may be mis-identified or even completely missed, as particle identification algorithms at hadron collider experiments generally assume signatures appropriate for SM particles. Additionally, some HSCPs may combine with SM particles to form composite objects. Interactions of these composite objects with the detector may change their constituents and possibly their electric charge, further limiting the ability of standard algorithms to identify them.

For HSCP masses greater than $100 \mathrm{GeV}$, a significant fraction of particles produced at the LHC have $\beta$ values less than 0.9 . These HSCPs can be identified by their longer time-of-flight (TOF) to outer detectors (muon system (MS) and/or calorimeters) or their anomalous amount of energy loss $(\mathrm{d} E / \mathrm{d} x)$. The $\mathrm{d} E / \mathrm{d} x$ of a particle depends on both its electric charge and its $\beta$. The dependence of $\mathrm{d} E / \mathrm{d} x$ on these variables is described by the Bethe-Bloch formula. In the momentum range of interest $(10-1000 \mathrm{GeV})$, SM charged particles have a relatively flat ionization energy loss and $\beta$ values very close to one. Searching for candidates with long TOF or large $\mathrm{d} E / \mathrm{d} x$ gives sensitivity to massive particles with $|Q|=1 e$ and particles with $|Q|>1 e$. On the other hand, searching for candidates with lower $\mathrm{d} E / \mathrm{d} x$ yields sensitivity to high-momentum particles with $|Q|<1 e$.

Details and results of the analyses carried out by the two collaborations are reported in the following sections.

\section{Multi-charged particles in ATLAS}

The search for heavy, long-lived, multi-charged particles (MCPs) traversing the ATLAS detector leaving a track in the inner tracking detector (ID), and producing a signal in the MS, is based on $20.3 \mathrm{fb}^{-1}$ of $\sqrt{s}=8 \mathrm{TeV}$ pp collisions data [11]. The search is performed in the MCP mass range of $50-1000 \mathrm{GeV}$, for electric charges $|Q|=z e$, with the charge numbers $z=2,3,4,5$, and 6. A purely electromagnetic coupling, proportional to the electric charge of the MCPs, is assumed for the production model: MCPs are produced in pairs via the Drell-Yan (DY) process with only photon exchange included. Events collected with a single-muon trigger are considered for the search. An additional calorimeter-based trigger with missing transverse momentum is employed to recovers some of the events missed by the single-muon trigger. The signal selection relies on the characteristic signature of a MCP in ATLAS. MCPs are highly ionising, and thus leave an abnormally large ionisation signal, $\mathrm{d} E / \mathrm{d} x$. Estimates of $\mathrm{d} E / \mathrm{d} x$ are evaluated for the pixel, transition radiation tracker (TRT) and MS precision chamber (MDT) sub-detector systems. Discriminators in the TRT are used to compare the signal from a straw tube with low and high thresholds (HT) using the TRT front-end electronics. The HT is designed to discriminate between energy depositions from transition radiation photons and the energy loss of minimum ionising particles. MCPs would 
produce a large number of HT hits along their trajectories due to their high level of ionisation. The background (manly mis-reconstructed high transverse moment $\left(p_{T}\right)$ muons) is estimated with data driven techniques. No signal candidate events are found and exclusion limits are computed. The measurement excludes the DY model of MCP pair-production over wide ranges of tested masses. Figure 1 Left shows the observed 95\% CL cross-section limits as a function of mass for the five different charges. At the lowest mass values the cross-section limit ranges from $7 \mathrm{fb}$ for $z=2$ to $1.4 \mathrm{pb}$ for $z=6$. For the DY model, the cross-section limits can be transformed into mass exclusion regions from $50 \mathrm{GeV}$ up to limits of $660,740,780,785$, and $760 \mathrm{GeV}$ for charge numbers $z=2$, $3,4,5$, and 6 , respectively. This result is similar to that obtained by the CMS collaboration (see Section 5).
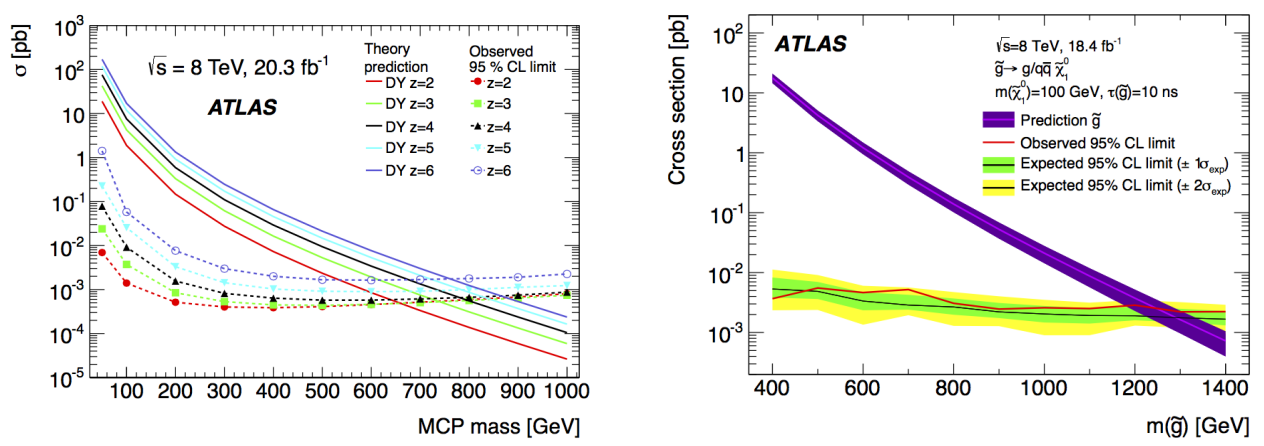

Figure 1: Left: ATLAS observed 95\% CL cross-section upper limits and theoretical cross-sections as functions of the MCPs mass for values of charge number $z$ between 2 and 6 [11]. Right: ATLAS upper limits on the production cross section as a function of mass for metastable gluino R-hadrons, with lifetime $\tau=10 \mathrm{~ns}$, decaying into $g / q \tilde{q}$ plus a light neutralino of mass $100 \mathrm{GeV}$ [12].

\section{Stable heavy charged particles in ATLAS}

Searches for heavy, long-lived, charged particles are performed using a data sample of 19.1 $\mathrm{fb}^{-1}$ of pp collisions at $\sqrt{s}=8 \mathrm{TeV}$ [12]. When travelling with a speed considerably slower than the speed of light, charged particles can be identified and their mass $(m)$ determined from their measured speed $(\beta)$ and momentum $(p)$, using the relation $m=p / \beta \gamma$, where $\gamma$ is the relativistic Lorentz factor. Three different searches have been carried out, using TOF in calorimeter and MS to measure $\beta$ and $\mathrm{d} E / \mathrm{d} x$ in ID, to measure $\beta \gamma$. Long-lived charged sleptons and charginos would interact like muons, releasing energy by ionisation as they pass through the detector: the search uses both the ID and the MS measurements. Coloured long-lived particles would hadronise forming R-hadrons, bound states of a gluino or squark with light SM quarks or gluons'. They may emerge as charged or neutral states from the pp collision and be converted to a state with a different charge by interactions with the detector material, and thus arrive as neutral, charged or doubly charged particles in the MS. Searches for R-hadrons are performed following two different approaches: using all available detector information, or disregarding all information from the MS. All searches are based on events collected with a single-muon trigger or a missing transverse momentum trigger. The background for all searches is almost entirely composed of high transverse momentum muons with mis-measured $\beta$ and/or large ionization: a data driven estimation has been used. No excess is 
observed above the estimated background and limits are placed on the mass of long-lived particles in various supersymmetric models. Long-lived tau sleptons in models with gauge-mediated symmetry breaking (GMSB) are excluded up to masses between 440 and $385 \mathrm{GeV}$ for $\tan \beta$ between 10 and 50, with a $290 \mathrm{GeV}$ limit in the case where only direct tau slepton production is considered. In the context of simplified LeptoSUSY models, where sleptons are stable and have a mass of 300 $\mathrm{GeV}$, squark and gluino masses are excluded up to a mass of 1500 and $1360 \mathrm{GeV}$, respectively. Directly produced charginos, in simplified models where they are nearly degenerate to the lightest neutralino, are excluded up to a mass of $620 \mathrm{GeV}$. R-hadrons, composites containing a gluino, bottom squark or top squark, are excluded up to a mass of 1270,845 and $900 \mathrm{GeV}$, respectively, using the full detector. R-hadrons are also excluded up to a mass of 1260, 835 and $870 \mathrm{GeV}$ using an approach disregarding information from the MS.

A search for charged, heavy, long-lived particles using ID measurement only has been also carried out, based on a data sample corresponding to an integrated luminosity of $18.4 \mathrm{fb}^{-1}$ of pp collisions at $\sqrt{s}=8 \mathrm{TeV}$ [13]. Measuring heavy, long-lived particles through their track parameters in the vicinity of the interaction vertex provides sensitivity to metastable particles with lifetimes from $0.6 \mathrm{~ns}$ to $30 \mathrm{~ns}$. This measurement does not depend on the way the long-lived particles interact in the dense calorimeter material nor on the decay mode. It can therefore address many different models of New Physics, especially those predicting the production of metastable heavy particles with O(ns) lifetime at LHC energies, such as mini-split SUSY or anomaly-mediated supersymmetry breaking (AMSB). The mass of the particles can be obtained by fitting their specific energy loss and momentum to an empirical Bethe-Bloch distribution [13]. The search is based on events collected with missing transverse momentum trigger. No significant deviation from the data driven SM background estimate is observed, and lifetime-dependent upper limits on R-hadrons (see Figure 1 Right) and chargino production are set. Gluino R-hadrons with $10 \mathrm{~ns}$ lifetime and masses up to $1185 \mathrm{GeV}$ are excluded at $95 \% \mathrm{CL}$, and so are charginos with $15 \mathrm{~ns}$ lifetime and masses up to $482 \mathrm{GeV}$.

\section{Search for monopoles and stable particles with high electric charges in ATLAS}

A search for highly charged particles stopping in the electromagnetic calorimeter, produced in proton-proton collisions at $8 \mathrm{TeV}$ centre-of-mass energy has been performed based on an integrated luminosity of $7.0 \mathrm{fb}^{-1}$ at $\sqrt{s}=8 \mathrm{TeV}$ [14]. Candidate events were selected by exploiting the measured ionisation in the TRT detector (HT hits) and the shape of the energy deposition in the EM calorimeter. Such features have also been used to design a dedicated trigger algorithm. No events were observed in data in the signal region over the estimated data-driven background. A model-independent upper limit on the production cross-section of $0.5 \mathrm{fb}$ was obtained for signal particles with magnetic charge in the range $0.5 g_{D} \leq|g| \leq 2.0 g_{D}$, where $g_{D} \sim 68.5 e$ is the Dirac charge, and electric charge in the range $20 \leq z \leq 60$ with masses between $200 \mathrm{GeV}$ and $2500 \mathrm{GeV}$. Assuming DY pair production of spin-1/2 and spin- 0 charged massive particles, upper limits on the production cross-section were obtained for $0.5 g_{D} \leq|g| \leq 1.5 g_{D}$ and $20 \leq z \leq 60$ and masses up to $2500 \mathrm{GeV}$ (see Figure 2). 

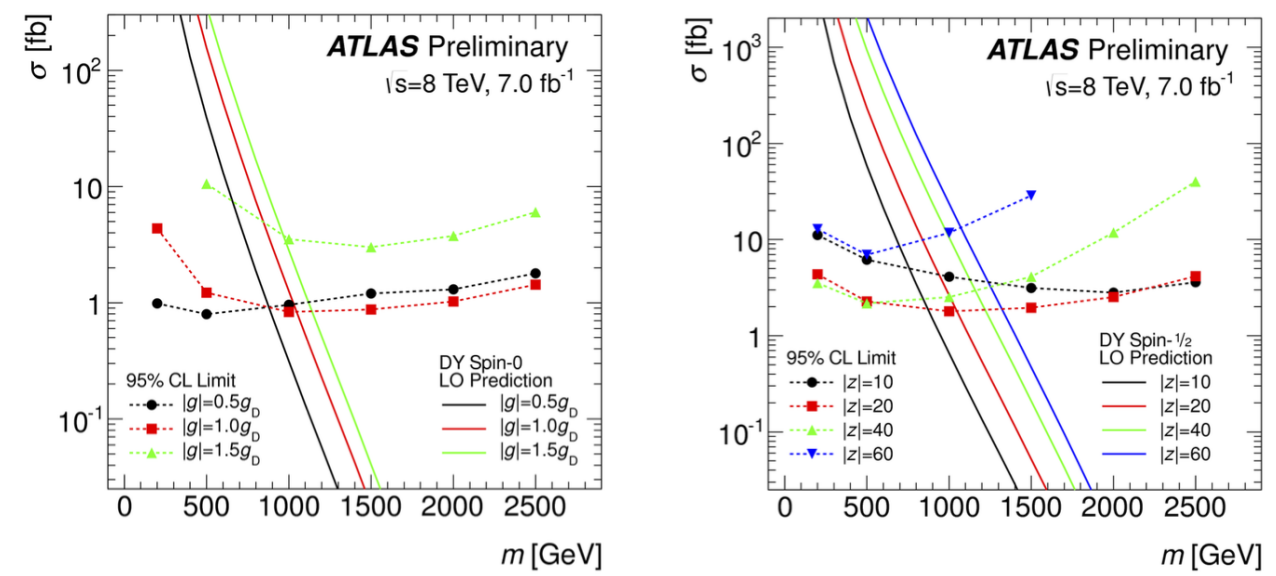

Figure 2: ATLAS cross-section upper limits at $95 \%$ CL for DY spin $1 / 2$ highly charged particles (Right) and spin 0 magnetic monopoles (Left) production as a function of particle mass in various scenarios (dashed lines with markers) [14]. Overlaid on the plots are the leading-order cross-sections (solid lines).

\section{High mass stable charged particles in CMS}

Five complementary analyses have been performed using data recorded at collision energies of 7 and $8 \mathrm{TeV}$ corresponding to an integrated luminosity of 5.0 and $18.8 \mathrm{fb}^{-1}$, respectively: a search with only the ID (track reconstructed only in ID and $\mathrm{d} E / \mathrm{d} x$ measurement), a search with both the ID and the MS (track reconstructed in ID and MS and $\mathrm{d} E / \mathrm{d} x$ and TOF measurements), a search with only the MS (track reconstructed only in MS and TOF measurement), a search for low ionizing particles (track reconstructed only in ID), and a search for tracks with very large ionization energy loss (track reconstructed in ID and MS) [15]. All searches are based on events collected with a single-muon trigger or a missing transverse momentum trigger. No significant excess is observed over the estimated data-driven background in any of the analyses. Limits on cross sections are presented for models with the production of gluinos, scalar tops, and staus, and for DY like production of fractionally, singly, and multiply charged particles. The models for R-hadrons include a varying fraction $f$ of $\tilde{g}$-gluon production and two different interaction schemes leading to a variety of non-standard experimental signatures. Gluino masses below 1322 and $1233 \mathrm{GeV}$ are excluded for $f=0.1$ in the cloud interaction model and the charge-suppressed model, respectively. For $f=0.5$ (1.0), gluino masses below 1276 (1250) GeV are excluded. For stop production, masses below 935 (818) GeV are excluded for the cloud (charge-suppressed) models. In addition, these analyses exclude masses below 500 (339) $\mathrm{GeV}$ for the direct+indirect (direct only) production. DY like signals with $|Q|=e / 3,2 e / 3,1 e, 2 e, 3 e, 4 e, 5 e, 6 e, 7 e$, and $8 e$ are excluded with masses below $200,480,574,685,752,793,796,781,757$, and $715 \mathrm{GeV}$ (see e.g Figure 3 Left), respectively. The tabulation of the relevant signal acceptance has been used to set stringent limits on the long-lived lepton-like sector of the phenomenological minimal supersymmetric standard model (pMSSM) and the AMSB model [16]. In the pMSSM parameter sub-space considered, 95.9\% of the points predicting charginos with a lifetime of at least $10 \mathrm{~ns}$ are excluded. Charginos with a lifetime greater than $100 \mathrm{~ns}$ and masses up to about $800 \mathrm{GeV}$ in the AMSB model are also excluded, see Figure 3 Right. The method can also be used to set constraints on other models [17]. 

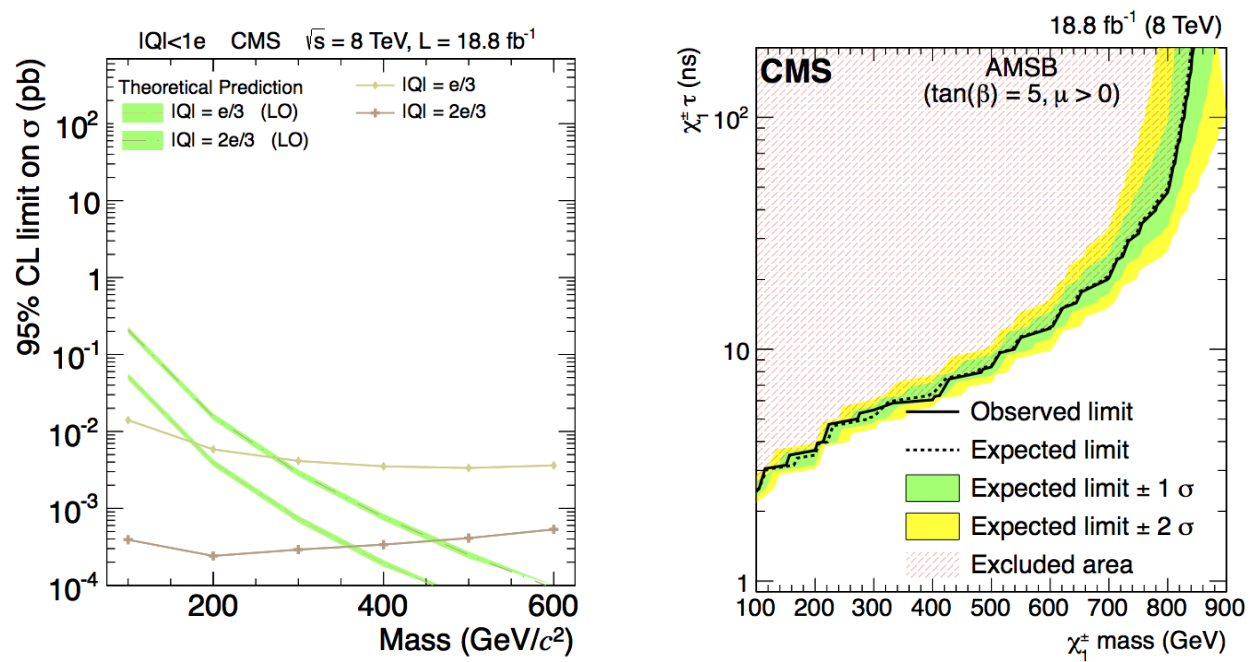

Figure 3: Left: CMS upper cross section limits at 95\% CL on fractionally charged particles for the analysis based on data at $\sqrt{s}=8 \mathrm{TeV}$ [15]. Right: CMS observed and expected excluded region on the chargino mass and lifetime parameter space in the context of the model with $\tan \beta=5$ and $\mu \geq 0$ (the excluded region is indicated by the hatched area) [16].

\section{References}

[1] M. Drees and X. Tata, Phys. Lett. B 252 (1990) 695.

[2] M. Fairbairn et al., Phys. Rept. 438 (2007) 1.

[3] C. W. Bauer et al., Phys. Lett. B 690 (2010) 280.

[4] J. S. Schwinger, Phys. Rev. 144 (1966) 1087.

[5] A. Kusenko and M. E. Shaposhnikov, Phys. Lett. B 418 (1998) 46.

[6] D. Fargion, M. Khlopov, and C. A. Stephan, Class. Quant. Grav. 23 (2006) 7305.

[7] B. Koch, M. Bleicher, and H. Stoecker, J. Phys. G 34 (2007) S535.

[8] J. Kang, P. Langacker, and B. D. Nelson, Phys. Rev. D 77 (2008) 035003.

[9] The ATLAS Collaboration, JINST 3 (2008) S08003.

[10] The CMS Collaboration, JINST 3 (2008) S08004.

[11] The ATLAS Collaboration, Eur. Phys. J. C 75 (2015) 362.

[12] The ATLAS Collaboration, JHEP 01 (2015) 068.

[13] The ATLAS Collaboration, Eur. Phys. J. C (2015) 75:407.

[14] The ATLAS Collaboration, CERN-PH-EP-2015-174 to be submitted to Phys. Rev. D.

[15] The CMS Collaboration, JHEP 07 (2013) 122.

[16] The CMS Collaboration, Eur. Phys. J. C 75 (2015) 325.

[17] The CMS Collaboration, Supplementary material from constraints on the pMSSM, AMSB model and on other models from the search for long-lived charged particles in $\sqrt{s}=8 \mathrm{TeV}$ pp collisions, (2014), deposited at http://hepdata.cedar.ac.uk/view/ins1343509. 